\title{
Colonization and Development of the Fecal Microflora of South China Tiger Cubs (Panthera tigris amoyensis) by Sequencing of the 165 rRNA Gene
}

\author{
Yanfa Sun ${ }^{a, b}, c$ Jie Yao ${ }^{a}$ Min Zhang ${ }^{a, b, c}$ Tengteng Chen ${ }^{d}$ Weihua $\mathrm{Xu}^{\mathrm{a}} \mathrm{b}, \mathrm{c}$ \\ Wenyuan Fu ${ }^{d}$ Qiong Wu ${ }^{a, b, c}$ Yan Li ${ }^{a, b, c}$ Xingxing Chen ${ }^{a, b, c}$ Yuting Zhu ${ }^{a}$ \\ Xuemei Zhang ${ }^{a} \quad$ Lingyu Liu $^{a}$ Donghong Chen ${ }^{a}$ Zhenyuan Wang ${ }^{a}$ \\ Zhangjing You ${ }^{a}$ Xuebing Zhang ${ }^{a}$ Yi Liu $^{a}$ Kaixiong Lin ${ }^{d}$ Weiming Lin ${ }^{a}$ b, c \\ ${ }^{a}$ College of Life Science, Longyan University, Longyan, China; ${ }^{b}$ Fujian Provincial Key Laboratory for the Prevention \\ and Control of Animal Infectious Diseases and Biotechnology, Longyan, China; ${ }^{\circ}$ Key Laboratory of Preventive \\ Veterinary Medicine and Biotechnology (Longyan University), Longyan, China; ${ }^{\mathrm{d}}$ Fujian Meihuashan South China \\ Tiger Breeding Institute, Longyan, China
}

\section{Keywords}

South China tiger · Panthera tigris amoyensis · $16 \mathrm{~S}$ rRNA gene $\cdot$ Fecal microflora

\begin{abstract}
Postnatal colonization and development of the gut microbiota is linked to health and growth. A comprehensive understanding of the postnatal compositional changes and development of the microbial community is helpful to understand the gut health and improve the survival rate of South China tiger cubs (Panthera tigris amoyensis). Fecal samples from three tiger cubs were collected on the day of birth in 2018 (June 17-21 [G0], July 18 [G1], July 31 [G2], and August 7 [G3]). The 16S rRNA genes of the fecal microflora were sequenced. Results showed that 38 phyla, 58 classes, 134 orders, 272 families, and 636 genera of bacteria from 3,059 operational taxonomic units were identified from 12 fecal sam-
\end{abstract}

ples. The diversity and abundance of species of group G0 were significantly higher $(p<0.05$ or 0.01$)$ than those of groups $\mathrm{G} 2$ and $\mathrm{G} 3$. The predominant phylum was Proteobacteria in groups G0 and G1 (38.85\% and 48\%, respectively) and Firmicutes in groups G2 and G3 (71.42\% and 75.29\%, respectively). At the phylum level, the abundance of Deinococcus-Thermus was significantly decreased in groups G1, $\mathrm{G} 2$, and $\mathrm{G} 3$ as compared to group $\mathrm{G} 0(p<0.05)$, while that of Firmicutes was significantly increased in groups $\mathrm{G} 2$ and $\mathrm{G} 3$ $(p<0.05)$. At the genus level, the abundance of Faecalibacterium, Ralstonia, and unidentified Rickettsiales was significantly decreased in groups G1, G2, and G3 as compared with group G0 ( $p<0.05)$, while that of Pseudomonas was significantly decreased in groups $\mathrm{G} 2$ and G3 ( $p<0.05)$. The composition and structure of fecal microbiota of South China tiger cubs changed after birth.

(C) 2021 The Author(s) Published by S. Karger AG, Basel karger@karger.com www.karger.com/mip

Karger $\stackrel{\text { ' }}{5}$

BOPEN ACCESS
(C) 2021 The Author(s)

Published by S. Karger AG, Basel

This is an Open Access article licensed under the Creative Commons Attribution-NonCommercial-4.0 International License (CC BY-NC) (http://www.karger.com/Services/OpenAccessLicense), applicable to the online version of the article only. Usage and distribution for commercial purposes requires written permission.
Correspondence to:

Kaixiong Lin, mhslkx@163.com

Weiming Lin,wmlin925@126.com 


\section{Introduction}

The South China tiger (Panthera tigris amoyensis), also known as the Xiamen or Amoy tiger, is the most threatened tiger subspecies [Qin and Nyhus, 2018]. Approximately 100 South China tigers have been raised in zoos or reserves [Jiang et al., 2016]. Under artificial feeding programs, due to low genetic diversity and inbreeding, the health and survival rate of tiger cubs are very poor [Chen et al., 2016]. Hence, increasing the survival rate is key to expand populations and prevent extinction.

The diversity and abundance of gut microbes are indicators of the health of both humans and animals [Alonso and Guarner, 2013; Lee and Hase, 2014; Long et al., 2018]. The gut microbiota plays a significant role in host metabolism and physiology [Nagpal and Yamashiro, 2015], which have been associated with health and growth [Feng et al., 2020]. Microbial colonization of the gastrointestinal tract begins in the early stages of life and the establishment of a complex and stable community is essential for optimal growth and development of the host [Marcolla et al., 2019]. The early establishment of the gut microbiota is influenced by environmental factors, as well as the mode of feed delivery, maternal diet, family structure, disease, and the use of medications [Nagpal and Yamashiro, 2015]. The microbes inhabiting the intestine are broadly divided into prokaryotes (bacteria and archaea), bacteriophages, viruses, and meiofauna, of which bacteria are the most abundant and comprise about $60 \%$ of the fecal mass [Andoh, 2016]. A previous study explored the impact of host intraspecies genetic variation, diet, and age on the intestinal microbiota of tiger cubs fed commercial milk in the Guangzhou Zoo [Jiang et al., 2020]. However, the effects of formulated milk on the intestinal microbiota of South China tiger cubs remained unclear.

Here, the succession of the fecal microbiota of tiger cubs was reported with the use of high-throughput sequencing technology to provide a foundation to further study the influence of formulated milk on the health and physiological regulation of tiger cubs.

\section{Results}

\section{Quality Assessment of Sequencing Data}

The results of data preprocessing statistics and quality control are shown in Table 1. An average of 84,416 raw reads and 80,111 clean reads were obtained for each sample. The quality of the sequencing data was $94.95 \%$ (online suppl. Table S3; for all online suppl. material, see www.

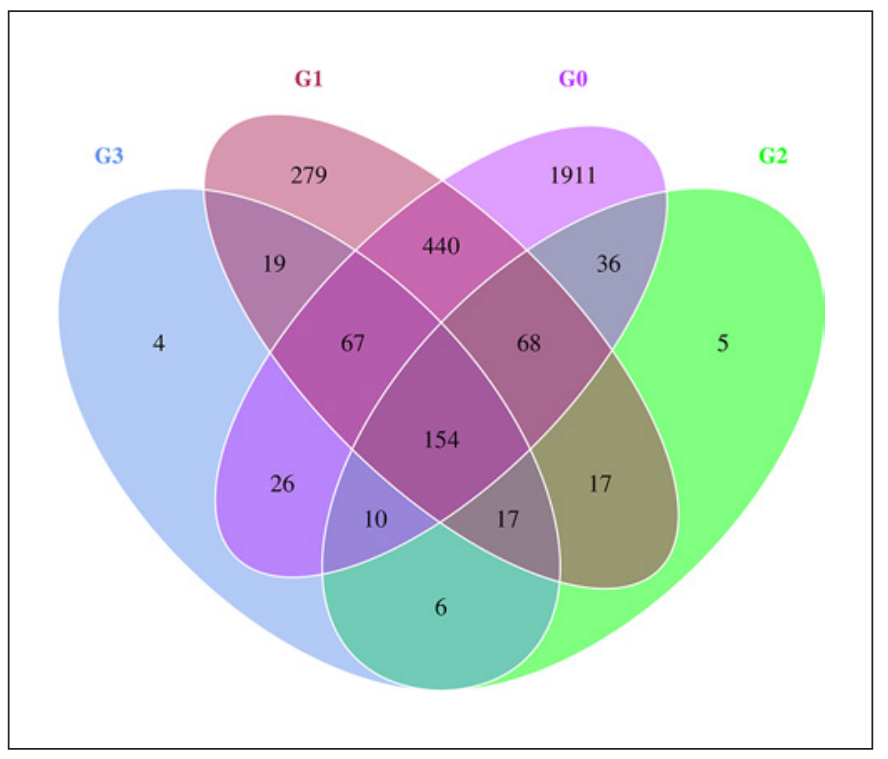

Fig. 1. Venn diagram showing number of OTUs among the four groups of fecal samples. The Venn diagram shows the number of OTUs among the fecal samples (G0, G1, G2, and G3).

karger.com/doi/10.1159/000518395). Sequences were clustered into operational taxonomic units (OTUs) based on a canonical clustering threshold of $97 \%$ identity [Stackebrandt and Goebel, 1994]. In this study, a total of 3,059 OTUs were acquired (Fig. 1 and online suppl. Table S4).

\section{Alpha Diversity Analysis}

Alpha diversity indices (Shannon, Simpson, Chao1, ACE, and Goods coverage) are shown in Table 1 and Figure 2. The Shannon and Simpson indices were used to measure species diversity. According to the Shannon and Simpson indices, there were significant differences in species diversity among groups G0, G2, and G3 $(p<0.01)$, as well as between groups G1 and G2 $(p<0.01)$. Meanwhile, according to the Shannon index, there were significant differences in the species diversity between groups G1 and G3 $(p<0.05)$. According to the Chaol and ACE indices, the abundance of species was significantly higher in group G0 than groups G2 and G3. The coverage of all sample libraries was greater than $99.9 \%$, indicating that the library size of this study was sufficient to represent the majority of bacteria.

\section{Bacterial Diversity and Relative Abundance}

In this study, a total of 38 phyla, 58 classes, 134 orders, 272 families, and 636 genera were identified in the fecal microbial communities of the 12 fecal samples (online suppl. Table S2). In groups G0 and G1, the predominant 
Table 1. Statistical analysis of the alpha diversity index

\begin{tabular}{llllll}
\hline Sample & Shannon & Simpson & Chao1 & ACE & Goods coverage \\
\hline D01 & 4.523 & 0.867 & 585.568 & 583.255 & 0.999 \\
D02 & 8.083 & 0.985 & $1,486.405$ & $1,489.770$ & 0.999 \\
D03 & 8.450 & 0.987 & $1,903.264$ & $1,875.906$ & 0.999 \\
D11 & 3.668 & 0.835 & 197.000 & 199.772 & 1.000 \\
D12 & 6.119 & 0.964 & $1,050.952$ & $1,052.133$ & 0.999 \\
D13 & 3.142 & 0.792 & 212.875 & 220.553 & 0.999 \\
D21 & 2.276 & 0.595 & 195.526 & 191.581 & 1.000 \\
D22 & 2.930 & 0.790 & 188.077 & 194.402 & 1.000 \\
D23 & 2.427 & 0.607 & 270.229 & 270.612 & 0.999 \\
D31 & 2.845 & 0.699 & 257.500 & 265.530 & 0.999 \\
D32 & 2.907 & 0.786 & 172.565 & 183.072 & 0.999 \\
D33 & 3.222 & 0.774 & 244.231 & 248.086 & 0.999 \\
\hline
\end{tabular}

phyla were Proteobacteria (38.85\% and $48 \%$ ), followed by Firmicutes (15.79\% and 29.07\%) and Cyanobacteria (21.47\%) in group G0 and Actinobacteria (12.12\%) in G1. The relative abundance of Firmicutes ranged from 15.79\% (G0) to $29.07 \%$ (G1) and was the dominant phyla in groups G2 (71.42\%) and G3 (75.29\%) (Table 2 and Fig. 3). Unidentified Cyanobacteria (21.80\%) and Sphingomonas $(9.09 \%)$ were the main species in group G0, while unidentified Enterobacteriaceae and unidentified Clostridiales were dominant in group G1 (15.96\% and $12.91 \%$, respectively). In addition, Megamonas and Streptococcus were the dominant genera with relative abundances of $42.19 \%$ and $24.87 \%$ in group $\mathrm{G} 2$, and $45.41 \%$ and $15.31 \%$ in group G3, respectively.

\section{Beta Diversity Analysis}

The fecal microbiome composition profiles of groups G0 and G1 were obviously separated from those of groups G2 and G3, as shown by the weighted UniFrac PCoA plot presented in Figure 4d. Hierarchical clustering UPGMA analysis showed that groups G0, G1, G2, and G3 were clearly individually clustered (Fig. 5).

\section{Significantly Altered Microbiota}

The species differences at the phylum and genus levels among the four groups by $t$ test analysis are shown in Figure 6. At the phylum level, as compared with group G0, the abundance of Deinococcus-Thermus was significantly decreased in groups G1, G2, and G3 ( $p<0.05$ ), while that of Firmicutes was significantly increased in groups G2 and G3 $(p<0.05)$. The abundance of Proteobacteria was significantly increased in group G2 $(p<0.05)$, while that of Fusobacteria was significantly decreased in groups G2 and G3 $(p<0.05)$. At the genus level, as compared with group G0, the abundances of Faecalibacterium, Ralstonia,
Table 2. Fecal bacterial composition among the four groups at the phylum level (\%)

\begin{tabular}{lllll}
\hline Phylum & G0 & G1 & G2 & G3 \\
\hline Firmicutes & 15.79 & 29.07 & 71.42 & 75.29 \\
Proteobacteria & 38.85 & 48.00 & 10.37 & 16.93 \\
Cyanobacteria & 21.47 & 0.43 & 0.07 & 0.11 \\
Actinobacteria & 7.92 & 12.12 & 10.94 & 2.89 \\
Bacteroidetes & 6.81 & 3.99 & 1.41 & 2.93 \\
Fusobacteria & 2.31 & 5.48 & 0.15 & 0.27 \\
Acidobacteria & 1.59 & 0.13 & 0.00 & 0.00 \\
Chloroflexi & 1.11 & 0.04 & 0.00 & 0.01 \\
Verrucomicrobia & 0.86 & 0.07 & 0.00 & 0.00 \\
Others & 2.54 & 0.47 & 0.01 & 0.01 \\
Unidentified bacteria & 0.75 & 0.20 & 5.62 & 1.55 \\
Firmicutes/Bacteroidetes & 2.32 & 7.29 & 50.65 & 25.70 \\
\hline
\end{tabular}

and unidentified Rickettsiales were significantly decreased in groups G1, G2, and G3 ( $p<0.05)$, while those of Pseudomonas were significantly decreased in groups G2 and G3 $(p<0.05)$. The abundance of Megamonas was significantly increased and that of unidentified Acidobacteria was significantly decreased in group G1 $(p<0.05)$.

\section{Discussion}

The gut microbiota of both humans and animals develop after birth and the composition will impact health in later life [Rodríguez et al., 2015; Laforest-Lapointe and Arrieta, 2017; Tanaka and Nakayama, 2017]. In humans, Enterobacteriaceae and Staphylococcus are the dominant gut microbiota after birth, while in the gut of infants, Bifidobacterium and some lactic acid bacteria are the main constituents. After weaning, the adult-type
Sun et al. 


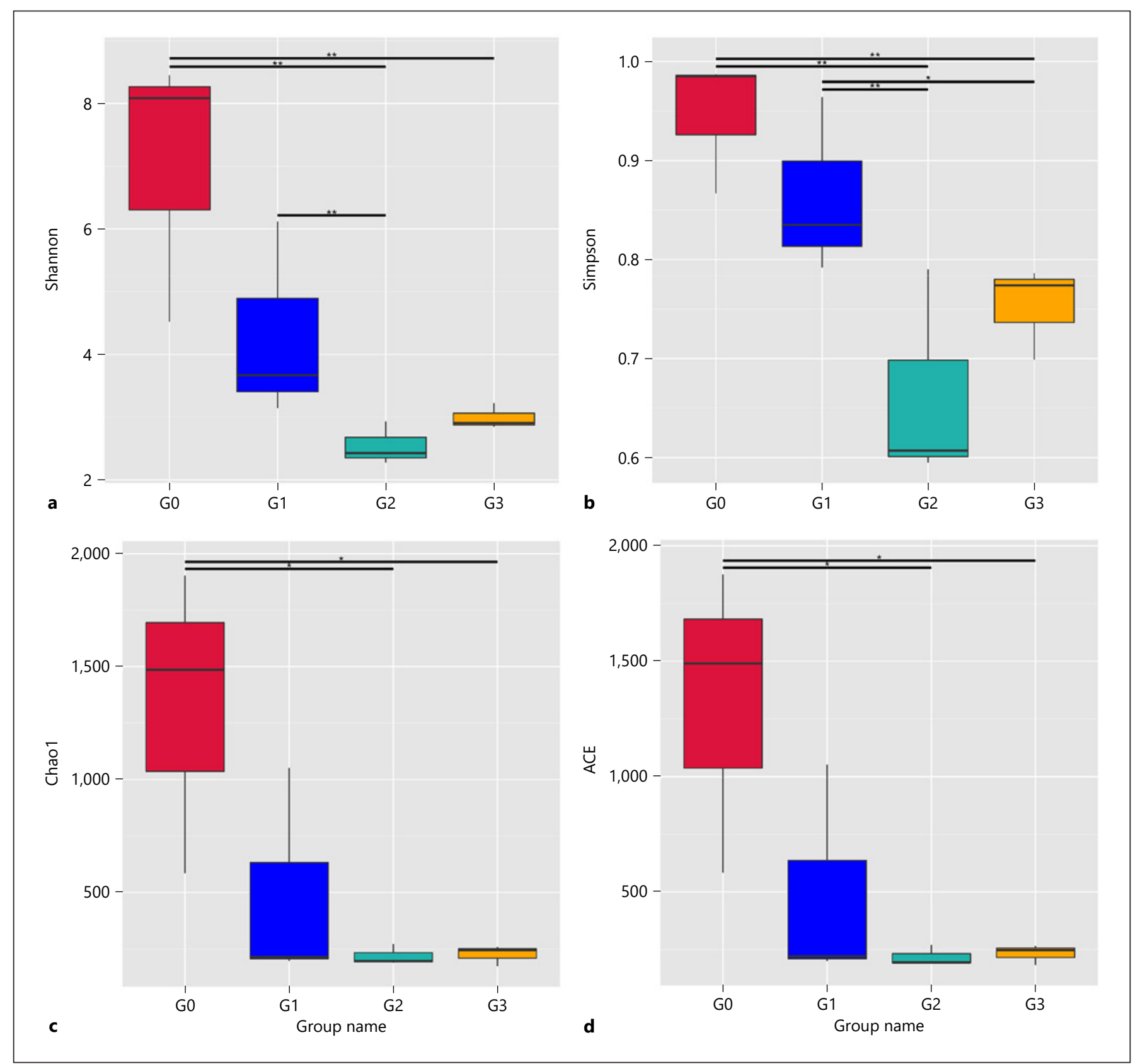

Fig. 2. Alpha diversity of fecal microbiota in the four groups. The alpha diversity of fecal microbiota in groups G0, G1, G2, and G3 by the Wilcoxon test. a Shannon; b Simpson; c Chao1; d ACE. * $p<0.05$; ${ }^{* *} p<0.01$.

microorganisms Bacteroides, Prevotella, Ruminococcus, Clostridium, and Veillonella colonize the infant intestines. By about the age of 3 years, a typical adult-like gut microbiota is established [Tanaka and Nakayama, 2017]. Previous studies have described the gut and fecal microbiota of the tiger [He et al., 2018a, b, c; Karmacharya et al., 2019]. However, the members of the microbial community of the tiger after birth remain unclear. Hence, the aim of the present study was to analyze changes in bacterial diversity and abundance in the fecal contents of tiger cubs.

After birth, the dominate phyla in the feces of tiger cubs are Proteobacteria, Firmicutes, and Cyanobacteria. At about postnatal day 50, the dominate phyla are Firmicutes and Proteobacteria. Proteobacteria account for $38.85 \%$ of the microbes in the gut of newborn cubs. Dur- 

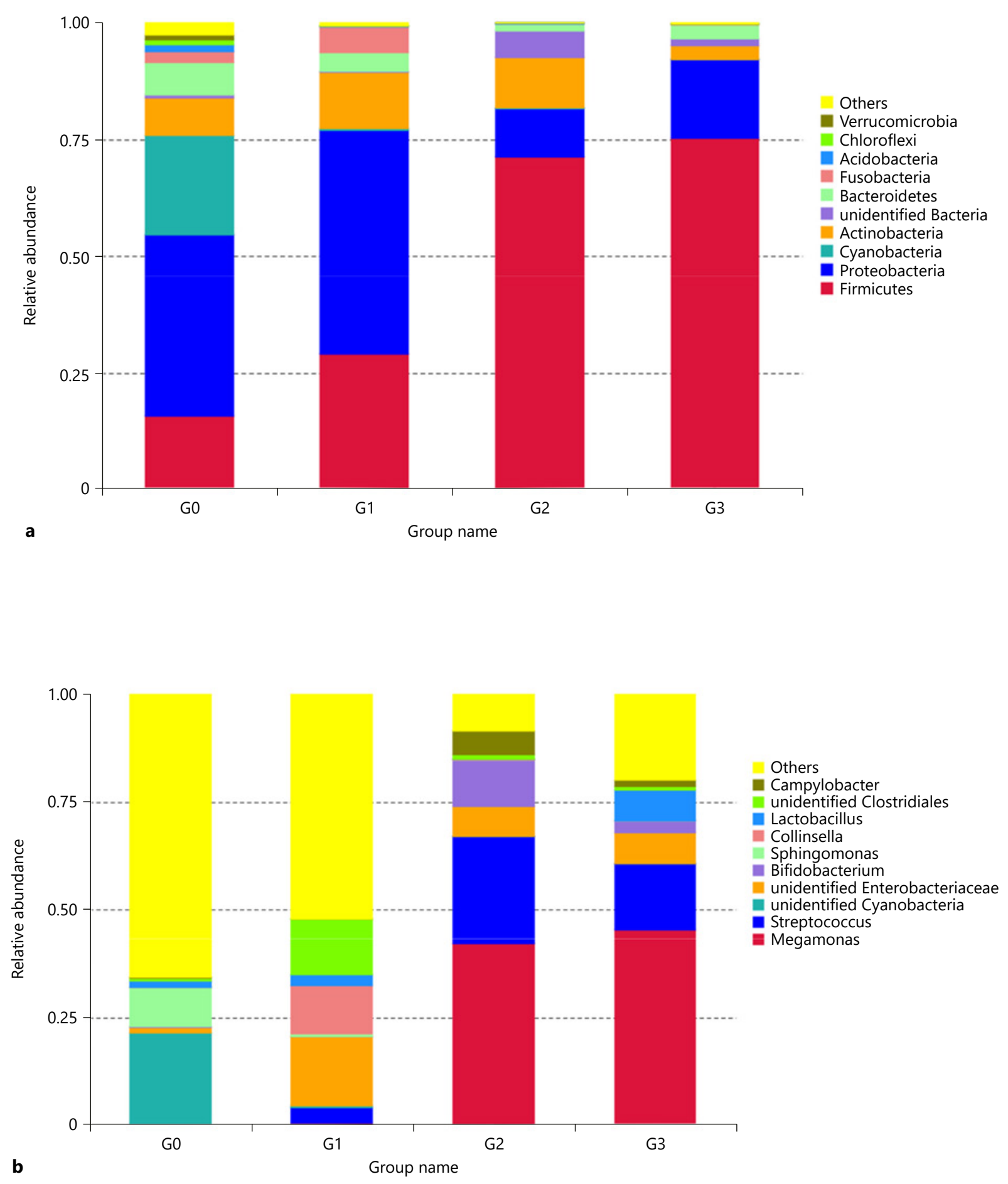


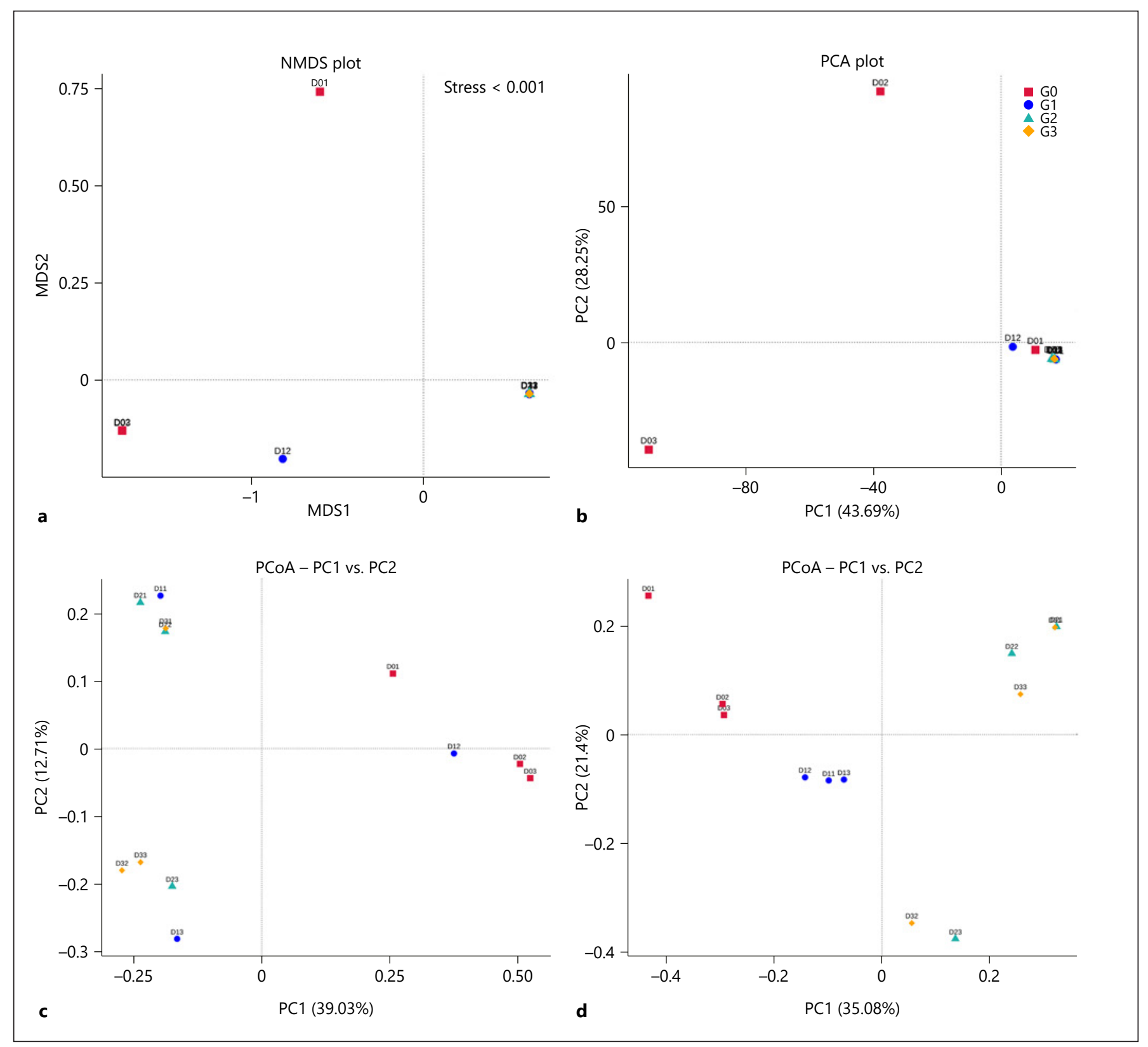

Fig. 4. NMDS (a), principal component analysis (b), and PCoA (c, d) of fecal bacterial community structures among the four groups. Different colors and shapes represent different groups. For PCoA, $\mathbf{c}$ and $\mathbf{d}$ were analyzed with weighted UniFrac distance and unweighted UniFrac distance, respectively.

ing initial colonization of the neonatal gut, Proteobacteria, which are facultative anaerobes and promote colonization of obligate anaerobes in the intestinal niche, are soon replaced by Firmicutes and Bacteroidetes, which are obligate anaerobes that dominate the gut microbiota of healthy adults [Shin et al., 2015]. At about postnatal day 50, the abundance of Proteobacteria had decreased to $16.93 \%$, while that of Firmicutes had increased to $75.29 \%$.
Firmicutes are the dominant phyla in the gut of healthy adults [Mariat et al., 2009]. The Firmicutes/Bacteroidetes ratio is commonly employed as a universal indicator of shifts in bacterial communities between infants and adults [Mariat et al., 2009; Zeineldin et al., 2018]. In the present study, the Firmicutes/Bacteroidetes ratio had increased with age due to the significant increase in the abundance of Firmicutes. 


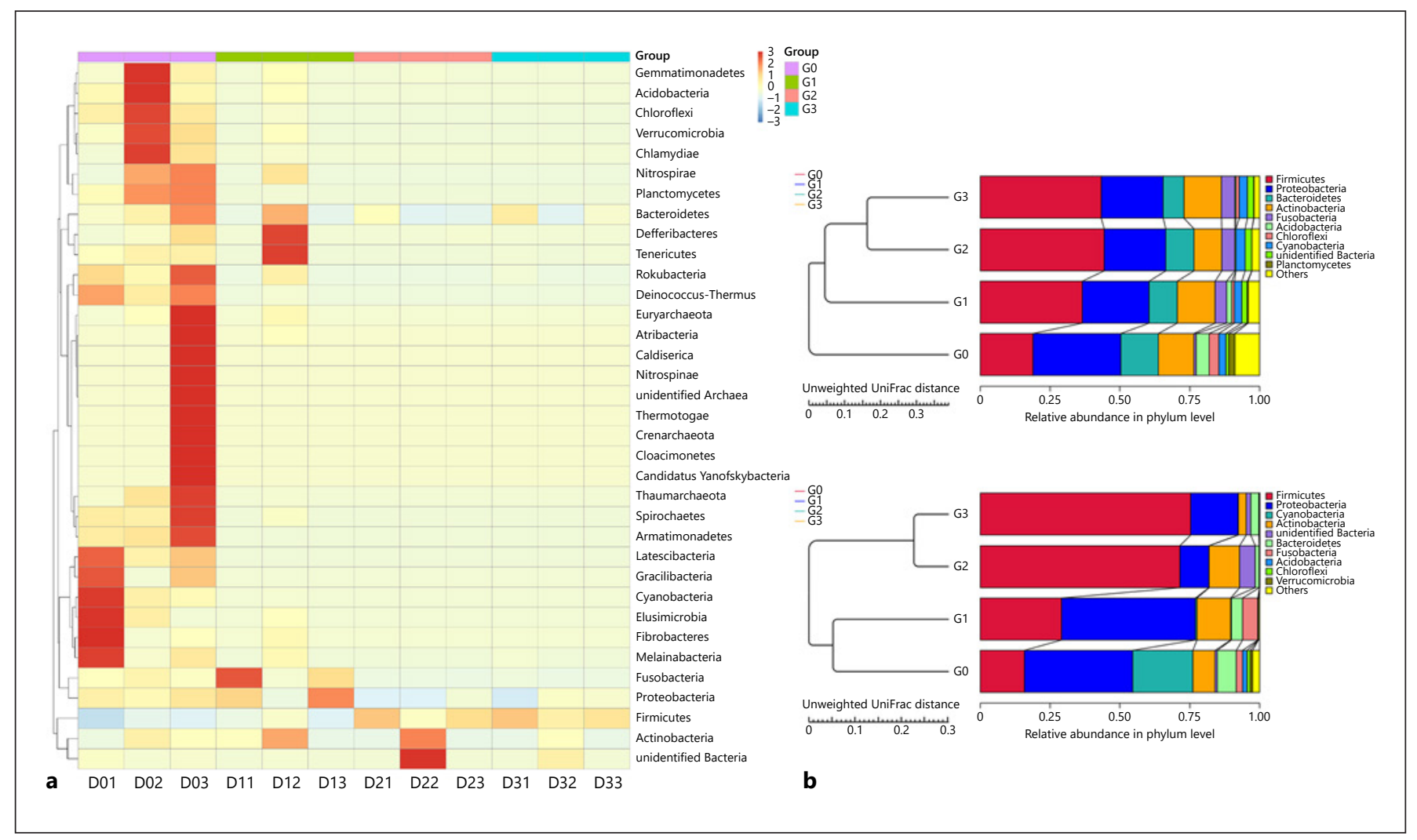

Fig. 5. Heat map of clustering for species richness (a) and UPGMA clustering trees with relative abundance at the phylum level (b). The heatmap of clustering for species richness (a) illustrates the bacterial distribution among different fecal samples. The bacterial phyla were clustered based on relative abundance. The $x$ axis represents each sample and the $y$ axis represents the relative percentage of each bacterial phyla. The $z$ value ( -3 to 3 ), as indicated by color intensity, is the relative abundance of the sample and the dif-

At birth, unidentified Cyanobacteria and Sphingomonas were the main microbes in the gut of tiger cubs. The phylum Cyanobacteria consists of gram-negative species, which are inferred to be one of the earliest branching bacterial lineages and have been found in the human gut [Di Rienzi et al., 2013]. A previous study reported that the fecal metagenome of captive Amur tigers had a lower abundance of Cyanobacteria [He et al., 2018b]. In the present study, the abundance of Cyanobacteria had decreased with age. Sphingomonas, which are gram-negative opportunistic human pathogens, are the predominant bacteria in fecal samples of pigs and chickens [Chien et al., 2011]. The Sphingomonas in feces efficiently degrades macromolecular organic pollutants, such as phenanthrene, which has carcinogenic activities in animals and induces an allergic response in the skin [Wu et al., 2010]. Megamonas and ference in the average relative abundance of all samples divided by the standard deviation of all samples in the classification. In $\mathbf{b}$, UPGMA clustering trees were generated with the weighted and unweighted UniFrac distance and then the trees were integrated with the relative abundances of species among all samples at the phylum level. The relative abundances of species at the phylum levels changed with the main composition phyla based on different UniFrac distances.

Streptococcus became the dominant genera with age. Megamonas, which can utilize fructooligosaccharides, is a predominant member of the family Veillonellaceae that has a potential impact on gastrointestinal health [Beloshapka et al., 2013]. In the family Streptococcaceae, the genus Streptococcus consists of gram-positive cocci that are considered to have certain advantages in the health of both commercial and domestic pigs [Huang et al., 2020]. Overall, the abundance of microorganisms in the gut of tiger cubs decreases with age, as in humans and other animals [Nagpal and Yamashiro, 2015; Rodríguez et al., 2015]. In the present study, the abundances of Faecalibacterium, Ralstonia, unidentified Rickettsiales, and Pseudomonas had significantly decreased at about postnatal day 50 .

In conclusion, the results of the present study revealed changes in microbial diversity and abundance in the gut 


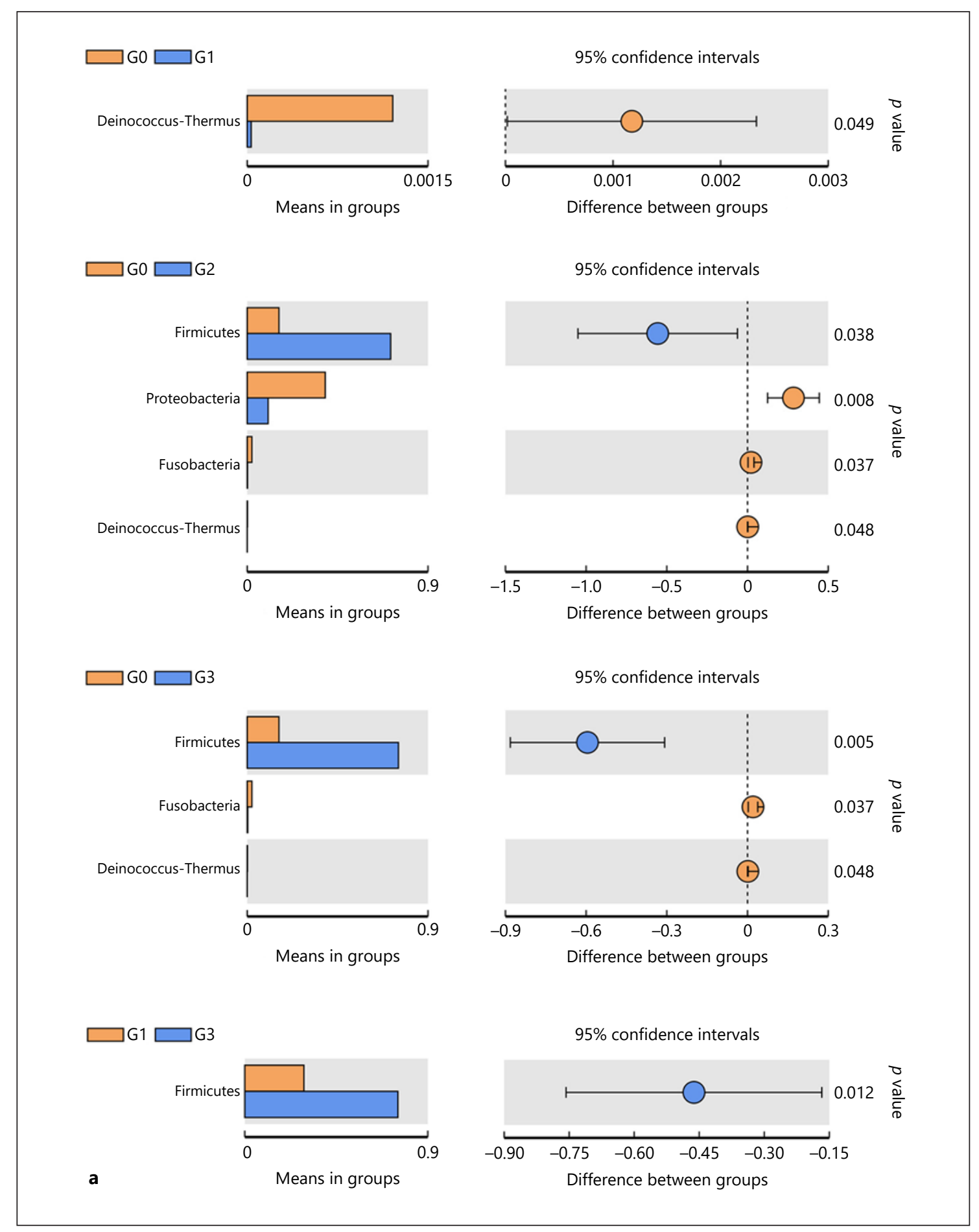

Fig. 6. Histogram of species differences at the phylum (a) and genus (b) levels among the four groups. The species differences at the phylum (a) and genus (b) levels in groups G0, G1, G2, and G3 were analyzed using the $t$ test. In each histogram, the left shows the abundances of differential species and each bar in the graph represents the mean values of species with significant differences in abundance among the groups. The figure on the right shows the confidence degree of inter-group differences. The leftmost end- point of each circle in the figure represents the lower limit of the 95\% confidence interval of the mean difference, and the rightmost endpoint of the circle represents the upper limit of the $95 \%$ confidence interval of the mean difference. The center of the circle represents the difference in the mean. The group represented by the color of the circle has a high mean value. At the far right of the results is the $p$ value of the inter-group significance test for differences in species.

(Figure continued on next page.) 
$\square$ G0 $\square$ G1
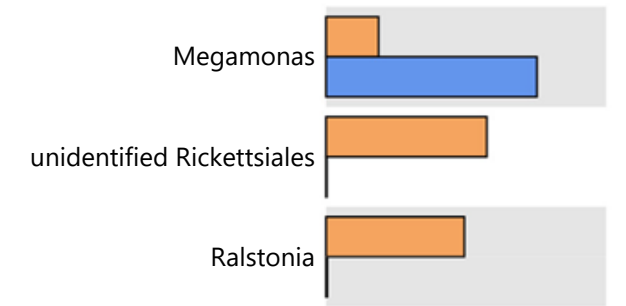

unidentified Acidobacteria

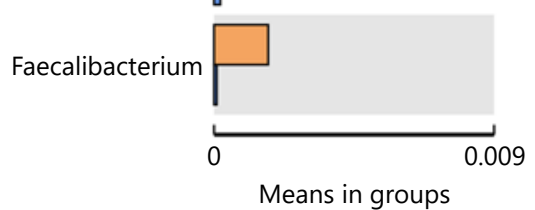

G0 $\square$ G2

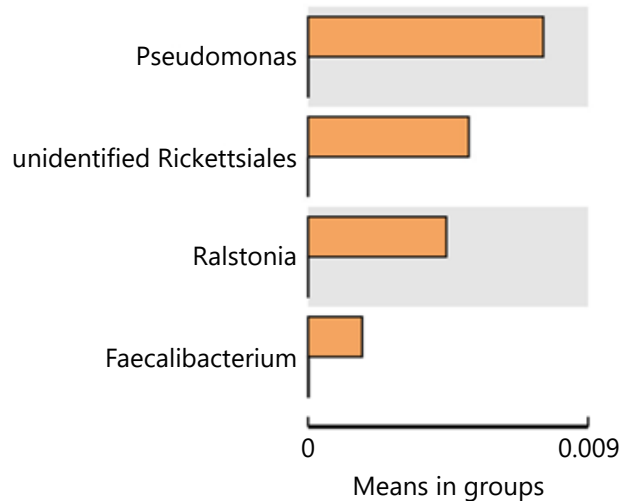

G0 $\square$ G3

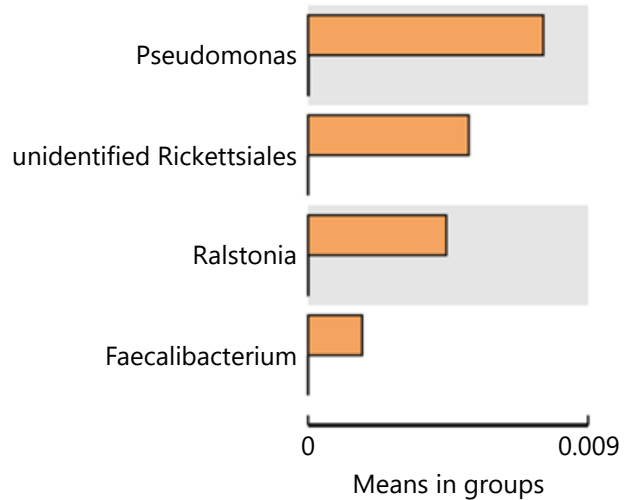

$95 \%$ confidence intervals

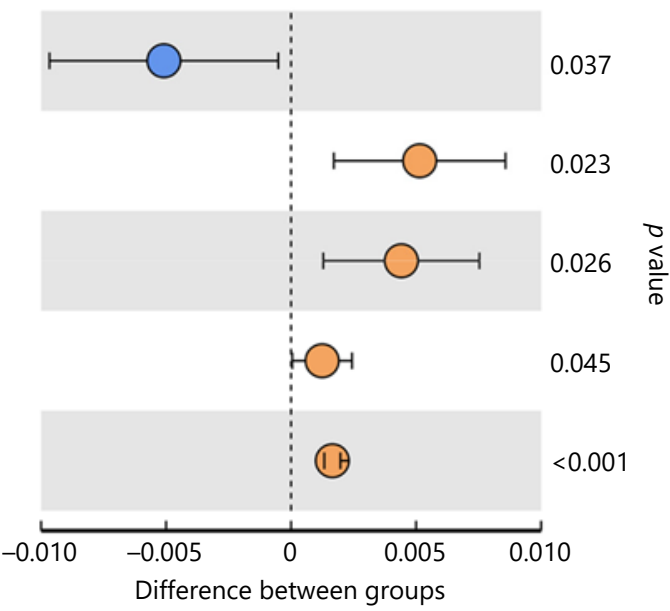

$95 \%$ confidence intervals

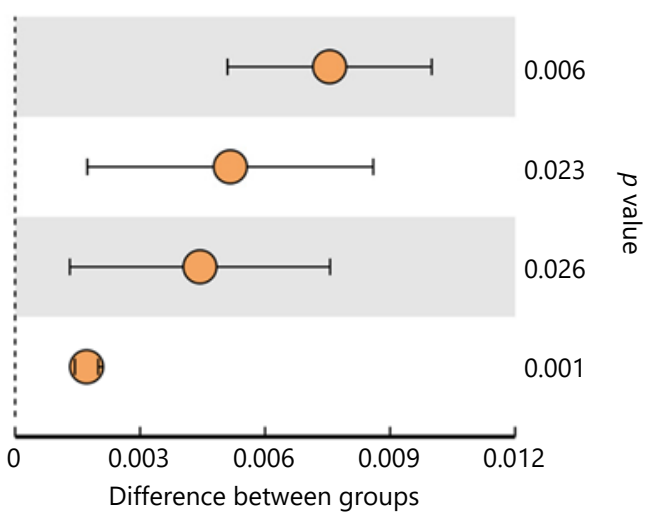

95\% confidence intervals

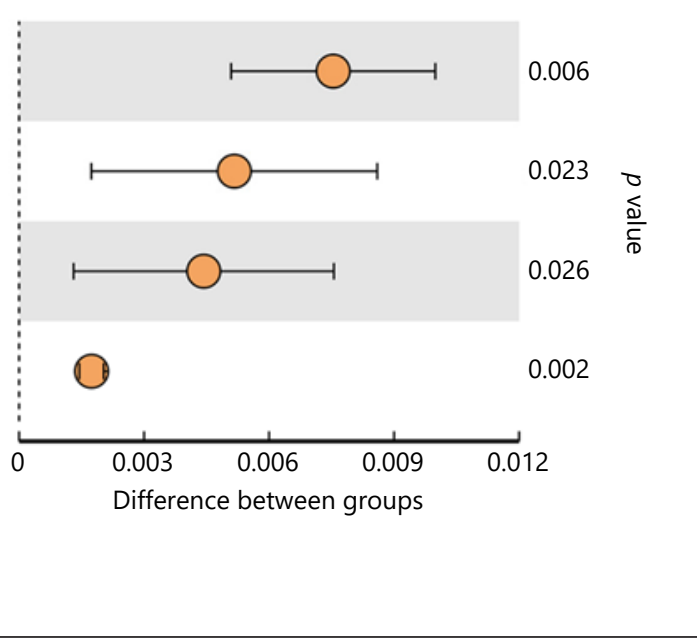


of tiger cubs after birth. Proteobacteria was the most predominant phylum in the gut of the South China tiger cubs at birth, which changed to Firmicutes at about postnatal day 50. Moreover, unidentified Cyanobacteria and Sphingomonas were the most abundant species in the gut of the tiger cub at birth, which were replaced by Megamonas and Streptococcus. The diversity and abundance of species had decreased with age. At the phylum level, the abundance of Deinococcus-Thermus had significantly decreased. Notably, at the genus level, the abundances of Faecalibacterium, Ralstonia, unidentified Rickettsiales, and Pseudomonas had significantly decreased. These findings provide further insight into the influence of diet on the health and physiological regulation of tiger cubs.

\section{Materials and Methods}

\section{Animals and Sample Collection}

Three South China tiger cubs from different litters born nearly on the same day at Fujian Meihuashan South China Tiger Breeding Institute (Fujian, China) were raised under the same environmental and nutritional conditions (online suppl. Tables S1 and S2). These cubs were abandoned by their mothers and fed artificially. The newborn cubs were raised in incubators at a constant temperature of $28^{\circ} \mathrm{C}$ for 1 week. Afterward, the temperature was maintained at about $25^{\circ} \mathrm{C}$ with relative humidity of $55-65 \%$. The incubators were cleaned and sanitized daily. After 3 weeks, the tiger cubs were moved into feeding chambers with similar environmental conditions as the incubators. During the artificial feeding period, the tiger cubs were in good health and defecated normally. The composition of formulated milk and feeding scheme are shown in online suppl. Tables S3 and S4, respectively. Fecal samples were collected in the morning on the day of birth in 2018 (June 17-21 [G0], July 18 [G1], July 31 [G2], and August 7 [G3]). Shortly after birth, the newborn tigers were stimulated to defecate and the feces were immediately collected aseptically, snap frozen in liquid nitrogen, and stored at $-80^{\circ} \mathrm{C}$ until assayed. Fecal sample collection was approved by Fujian Meihuashan South China Tiger Breeding Institute.

\section{DNA Extraction and 16S rRNA Gene Amplification}

Total bacterial genomic DNA from fecal samples was extracted using the cetyl trimethylammonium bromide method as described by Tilson et al. [2004]. DNA concentration and purity were monitored on $1 \%$ agarose gels, while quantity and quality were measured using a NanoDrop ${ }^{\mathrm{TM}}$ One spectrophotometer (Thermo Fisher Scientific, Waltham, MA, USA). Genomic DNA was diluted in sterile water to a concentration of $1 \mathrm{ng} / \mu \mathrm{L}$. The $\mathrm{V} 4$ region of the $16 \mathrm{~S}$ rRNA gene was amplified using the primer pair 528F (GCGGTAATTCCAGCTCCAA) and 706R (AATCCRAGAATTTCACCTCT). All PCR reactions were carried out in a $30-\mu \mathrm{L}$ reaction volume consisting of $15 \mu \mathrm{L}$ of Phusion ${ }^{\circledR}$ High-Fidelity PCR Master Mix (New England Biolabs, Ipswich, MA, USA), $0.2 \mu \mathrm{M}$ forward and reverse primers, and $10 \mathrm{ng}$ of template DNA. The thermal cycling conditions consisted of an initial denaturation step at $98^{\circ} \mathrm{C}$ for $1 \mathrm{~min}$, followed by 30 cycles of denaturation at $98^{\circ} \mathrm{C}$ for $10 \mathrm{~s}$, annealing at $50^{\circ} \mathrm{C}$ for
$30 \mathrm{~s}$, and elongation at $72^{\circ} \mathrm{C}$ for $30 \mathrm{~s}$, with a final extension step at $72^{\circ} \mathrm{C}$ for $5 \mathrm{~min}$.

The PCR products were mixed with the same volume of $1 \times$ loading buffer (containing SYBR green) and then separated by electrophoresis on a $2 \%$ agarose gel. The samples were pooled by mixing the PCR products at equidensity ratios and then purified with the GeneJET TM Gel Extraction Kit (Thermo Fisher Scientific).

\section{Library Preparation and Sequencing}

Sequence libraries were generated using the Ion Plus Fragment Library Kit 48 rxns (Thermo Fisher Scientific) in accordance with the manufacturer's instructions. The library quality was assessed with the use of a Qubit ${ }^{\circledR}$ 2.0 Fluorometer (Thermo Fisher Scientific). Then, the library was sequenced on an Ion S5 ${ }^{\mathrm{TM}} \mathrm{XL}$ platform and 400/600-base pair single-end reads were generated.

\section{Data Analysis}

Single-end reads were truncated by cutting off the barcodes and primer sequences. Quality filtering of the raw reads was performed under specific filtering conditions to obtain high-quality clean reads with the use of Cutadapt version 1.9.1 software [Martin, 2011]. The reads were compared to the reference Silva database [Quast et al., 2012] using the UCHIME algorithm [Edgar et al., 2011] to detect chimera sequences, which were then removed to obtain clean reads [Haas et al., 2011].

Sequence analysis was performed using Uparse version 7.0.1001 software [Edgar, 2013]. Sequences with $\geq 97 \%$ similarity were assigned to the same OTU. The Silva database was used based on the Mothur algorithm to annotate taxonomic information of each representative sequence of each OTU. In order to assess the phylogenetic relationships among different OTUs and the differences among the dominant species of different samples (groups), multiple sequence alignment was conducted using MUSCLE version 3.8.31 software [Edgar, 2004]. The abundance of each OTU was normalized using a standardized sequence number corresponding to the sample with the least sequences.

Analyses of the alpha and beta diversity values of the output normalized data were performed. Alpha diversity was applied to analyze the complexity of species diversity for a sample with the use of six indices (Observed-species, Chaol, Shannon, Simpson, ACE, and Good-coverage), as determined with the QIIME (version 1.7.0) open-source bioinformatics pipeline [Caporaso et al., 2010] and displayed with R software (version 2.15.3). Beta diversity analysis was used to evaluate differences in species complexity among the samples. The beta diversities of both weighted (quantitative) and unweighted (qualitative) UniFrac values were calculated with the use of QIIME software (version 1.7.0).

Cluster analysis was preceded by principal component analysis, which was applied to reduce the dimension of the original variables using the FactoMineR package and ggplot2 package in $\mathrm{R}$ software (version 2.15.3). Principal coordinate analysis (PCoA) was performed to obtain and visualize principal coordinates from complex, multidimensional data. A distance matrix of weighted or unweighted UniFrac values among samples was obtained and then transformed to a new set of orthogonal axes, by which the maximum variation factor was demonstrated by the first principal coordinate and the second by the second principal coordinate, and so on. PCoA analysis was conducted with the use of the WGCNA package, stat package, and ggplot 2 package in $\mathrm{R}$ software (version 2.15.3). The clustering of unweighted pair-group method with 
arithmetic means (UPGMA) was performed using QIIME software (version 1.7.0), as a type of hierarchical clustering method to interpret the distance matrix using average linkage values.

\section{Acknowledgements}

We wish to thank International Science Editing (http://www. internationalscienceediting.com) for editing of the manuscript.

\section{Statement of Ethics}

The study protocol was approved by the Ethics Committee of Longyan University (Longyan, Fujian province, China) (approval No. ECLU2018001).

\section{Conflict of Interest Statement}

The authors have no competing interests to declare.

\section{Funding Sources}

This study was supported by grants from the Fujian Provincial Natural Science Foundation of China (grant No. 2018J01460), Educational Research Project for Young and Middle-aged Teachers in Fujian Province of China (grant No. JAT160482), Key Natural Science Foundation for Young-aged Teachers of University in Fu- jian Province of China (grant No. JZ160482), Fujian Province Undergraduate Innovation and Entrepreneurship Training Program (201911312048), and the Special Fund for Local Science and Technology Development Guided by the Chinese Government (grant No. 2018L3011). The funding agencies had no role in the study design, data collection/analysis, decision to publish, or preparation of the manuscript.

\section{Author Contributions}

Yanfa Sun conceived, designed, and performed the experiments, analyzed the data, and authored and reviewed drafts of the paper; Weihua Xu prepared the formulated milk, collected and disposed of the fecal samples, and authored or reviewed drafts of the paper; Wenyuan Fu, Min Zhang and Tengteng Chen collected and disposed of the fecal samples, and authored or reviewed drafts of the paper; Kaixiong Lin and Weiming Lin conceived and designed the experiments, and authored or reviewed drafts of the paper; Jie Yao, Min Zhang, Qiong Wu, Yan Li, Xingxing Chen, Yuting Zhu, Xuemei Zhang, Lingyu Liu, Donghong Chen, Zhenyuan Wang, Zhangjing You, Xuebing Zhang, and Yi Liu performed the experiments, and authored and reviewed drafts of the paper. All authors have read and approved the final version of the manuscript.

\section{Data Availability Statement}

The raw data were deposited into the NCBI Sequence Read Archive (SRA) database (BioProject No. PRJNA609398).

\section{References}

Alonso VR, Guarner F. Linking the gut microbiota to human health. British Journal of Nutrition. 2013;109(S2):S21-S26.

Andoh A. Physiological role of gut microbiota for maintaining human health. Digestion. 2016; 93(3):176-81.

Beloshapka AN, Dowd SE, Suchodolski JS, Steiner JM, Duclos L, Swanson KS. Fecal microbial communities of healthy adult dogs fed raw meat-based diets with or without inulin or yeast cell wall extracts as assessed by 454 pyrosequencing. FEMS Microbiol Ecol. 2013; 84(3):532-41.

Caporaso JG, Kuczynski J, Stombaugh J, Bittinger $\mathrm{K}$, Bushman FD, Costello EK, et al. QIIME allows analysis of high-throughput community sequencing data. Nat Methods. 2010;7(5): 335.

Chen L, Li S, Lei S, Xie H. Study of the Young South China Tigers under the Condition of Artificial Feeding with US KMR Cat Milk. World Journal of Forestry. 2016;4(5):111-5.

Chien YC, Chen CJ, Lin TH, Chen SH, Chien YC Characteristics of microbial aerosols released from chicken and swine feces. J Air Waste Manag Assoc. 2011;61(8):882-9.
Di Rienzi SC, Sharon I, Wrighton KC, Koren O, Hug LA, Thomas BC, et al. The human gut and groundwater harbor non-photosynthetic bacteria belonging to a new candidate phylum sibling to Cyanobacteria. elife. 2013;2:e01102.

Edgar RC. MUSCLE: multiple sequence alignment with high accuracy and high throughput. Nucleic Acids Res. 2004;32(5):1792-7.

Edgar RC. UPARSE: highly accurate OTU sequences from microbial amplicon reads. Nat Methods. 2013;10(10):996.

Edgar RC, Haas BJ, Clemente JC, Quince C, Knight R. UCHIME improves sensitivity and speed of chimera detection. Bioinformatics. 2011;27(16):2194-200.

Feng L, Raman AS, Hibberd MC, Cheng J, Griffin NW, Peng Y, et al. Identifying determinants of bacterial fitness in a model of human gut microbial succession. Proc Natl Acad Sci U S A. 2020;117(5):2622-33.

Haas BJ, Gevers D, Earl AM, Feldgarden M, Ward DV, Giannoukos G, et al. Chimeric 16S rRNA sequence formation and detection in Sanger and 454-pyrosequenced PCR amplicons. Genome Res. 2011;21(3):494-504.
He F, Liu D, Zhai J, Zhang L, Ma Y, Xu Y, et al. Metagenomic analysis revealed the effects of goat milk feeding and breast feeding on the gut microbiome of Amur tiger cubs. Biochem Biophys Res Commun. 2018a;503(4):2590-6.

He F, Liu D, Zhang L, Zhai J, Ma Y, Xu Y, et al. Metagenomic analysis of captive Amur tiger faecal microbiome. BMC Vet Res. 2018b; 14(1):379.

He F, Zhai J, Zhang L, Liu D, Ma Y, Rong K, et al. Variations in gut microbiota and fecal metabolic phenotype associated with Fenbendazole and Ivermectin Tablets by 16S rRNA gene sequencing and LC/MS-based metabolomics in Amur tiger. Biochem Biophys Res Commun. 2018c;499(3):447-53.

Huang J, Zhang W, Fan R, Liu Z, Huang T, Li J, et al. Composition and functional diversity of fecal bacterial community of wild boar, commercial pig and domestic native pig as revealed by $16 \mathrm{~S}$ rRNA gene sequencing. Arch Microbiol. 2020;202:843-57.

Jiang H, Chen W, Su L, Huang M, Lin L, Su Q, et al. Impact of host intraspecies genetic variation, diet, and age on bacterial and fungal intestinal microbiota in tigers. Microbiologyopen. 2020 Jul;9(7):e1050. 
Jiang Z, Li L, Luo Z, Tang S, Li C, Hu H, et al. Evaluating the status of China's mammals and analyzing their causes of endangerment through the red list assessment. Biodiversity Science. 2016;24(5):552-67.

Karmacharya D, Manandhar P, Manandhar S, Sherchan AM, Sharma AN, Joshi J, et al. Gut microbiota and their putative metabolic functions in fragmented Bengal tiger population of Nepal. PLoS One. 2019;14(8):e0221868.

Laforest-Lapointe I, Arrieta MC. Patterns of early-life gut microbial colonization during human immune development: an ecological perspective. Front Immunol. 2017;8:788.

Lee WJ, Hase K. Gut microbiota-generated metabolites in animal health and disease. Nat Chem Biol. 2014;10(6):416-24.

Long SF, Xu YT, Pan L, Wang QQ, Wang CL, Wu $\mathrm{JY}$, et al. Mixed organic acids as antibiotic substitutes improve performance, serum immunity, intestinal morphology and microbiota for weaned piglets. Animal Feed Science and Technology. 2018;235:23-32.

Marcolla CS, Alvarado CS, Willing BP. Early life microbial exposure shapes subsequent animal health. Can J Anim Sci. 2019;99(4):661-77.
Mariat D, Firmesse O, Levenez F, Guimarăes V, Sokol H, Doré J, et al. The Firmicutes/Bacteroidetes ratio of the human microbiota changes with age. BMC Microbiol. 2009;9: 123.

Martin M. Cutadapt removes adapter sequences from high-throughput sequencing reads. EMBnet J. 2011;17(1):10-2.

Nagpal R, Yamashiro Y. Early-life gut microbial composition. J Pediatr Biochem. 2015;5(02): 041-050.

Qin Y, Nyhus PJ. Assessing factors influencing a possible South China tiger reintroduction: a survey of international conservation professionals. Environmental Conservation. 2018; 45(1):58-66.

Quast C, Pruesse E, Yilmaz P, Gerken J, Schweer $\mathrm{T}$, Yarza $\mathrm{P}$, et al. The SILVA ribosomal RNA gene database project: improved data processing and web-based tools. Nucleic Acids Res. 2012;41(D1):D590-6.

Rodríguez JM, Murphy K, Stanton C, Ross RP, Kober OI, Juge N, et al. The composition of the gut microbiota throughout life, with an emphasis on early life. Microb Ecol Health Dis. 2015;26(1):26050.
Shin NR, Whon TW, Bae JW. Proteobacteria: microbial signature of dysbiosis in gut microbiota. Trends Biotechnol. 2015;33(9):496-503.

Stackebrandt E, Goebel BM. Taxonomic note: a place for DNA-DNA reassociation and $16 \mathrm{~S}$ rRNA sequence analysis in the present species definition in bacteriology. Int J Syst Evol Microbiol. 1994;44(4):846-9.

Tanaka M, Nakayama J. Development of the gut microbiota in infancy and its impact on health in later life. Allergol Int. 2017;66(4):515-22.

Tilson R, Defu H, Muntifering J, Nyhus PJ. Dramatic decline of wild South China tigers Panthera tigris amoyensis: field survey of priority tiger reserves. Oryx. 2004;38(1):40-7.

Wu C, Yang F, Gao R, Huang Z, Xu B, Dong Y, et al. Study of fecal bacterial diversity in Yunnan snub-nosed monkey (Rhinopithecus bieti) using phylogenetic analysis of cloned 16S rRNA gene sequences. Afr J Biotechnol. 2010; 9(38):6278-89.

Zeineldin M, Aldridge B, Blair B, Kancer K, Lowe J. Impact of parenteral antimicrobial administration on the structure and diversity of the fecal microbiota of growing pigs. Microb Pathog. 2018;118:220-9. 